\title{
Downlink SINR distribution of linearly precoded multiuser MIMO systems
}

\author{
Zihuai Lin, Troels B. Sørensen, Preben E. Mogensen
}

\begin{abstract}
This paper derives mathematical expressions for the SINR distribution in systems with linearly precoded multiuser MIMO and frequency domain packet scheduling. The packet scheduler is able to exploit the available multiuser diversity in both time, frequency and spatial domains. Our analysis model is confined with 3GPP downlink transmission in which we specifically investigate the Single User (SU) and Multi-user (MU) Spatial Divsion Multiplexing (SDM) MIMO schemes. From the analytical results we find that the outage probability for systems using the SU-MIMO scheme is larger than the one for the MU-MIMO scheme. Also, in comparison to systems without precoding, linear precoding can improve the outage probability.
\end{abstract}

\section{INTRODUCTION}

Linear precoding for multiuser Multiple-Input Multiple-Output (MIMO) systems has attracted much attention recently due to the capacity enhancement ability at the system level. In the literature, most of the work focus on the design of the transmitter precoding matrix, e.g., $[1,2]$. In $[3,4]$, the interaction between packet scheduling and array antenna techniques is studied based on a system level simulation model. Analytical study on the interactions between multiuser diversity and spatial diversity is investigated in [5] with the focus on space time block coding. To the authors knowledge, theoretical analysis of linearly precoded multiuser Spatial Division Multiplexing (SDM) MIMO systems combined with Frequency Domain (FD) packet scheduling has not been studied so far.

This paper is about the analysis of the distribution of Signal to Interference plus Noise Ratio (SINR) for multiuser spatial multiplexing MIMO systems in combination with the base station based packet scheduler. The packet scheduler is used to exploit the available multiuser diversity in the time, frequency and spatial domains. While the method of analysis is quite general, and thus applicable to general packet based systems, in this work we base our study specifically on 3GPP UTRA Long Term Evolution (LTE) downlink [6] packet data transmission. In this specific setting we derive novel mathematical expressions for the SINR distribution in a number of cases. We will start by considering a few results for the instantaneous SINR distribution of 
the substreams, which we will subsequently use to derive the effective post scheduling SINR distributions

\section{SYSTEM MODEL}

In this section, linearly precoded multiuser MIMO schemes with packet scheduling are treated. The basic scheduling unit in UTRA LTE is regarded as the Physical Resource Block (PRB), which consists of a number of consecutive OFDM sub-carriers reserved during a fixed number of OFDM symbols. One PRB of 12 contiguous subcarriers can be configured for localized transmission in a sub-frame.

With the localized transmission scheme, two SDM schemes are now under investigation, they are Single User (SU) MIMO and Multi-User (MU) MIMO schemes. They differ in terms of the freedom allowed to the scheduler in the spatial domain [6]. For SU-MIMO scheme, only one single user can be scheduled per PRB. For MU-MIMO scheme, multiple users can be scheduled per PRB, one user for each substream per PRB.

The FD scheduling algorithm used in this work is the FD Proportional Fair (PF) [7] packet scheduling algorithm which is being investigated under LTE. With simplified assumptions similar to [8], i.e., user's fading statistics are independent identically distributed and there is (approximately) an linear relationship between the throughput and the SINR, the PF scheduling algorithm can be seen as a selection diversity process which selects the users with the best unified effective SINR. The unified effective SINR is defined as the equivalent single SINR which offers the same instantaneous (Shannon) capacity as a MIMO scheme with multiple streams, see $[9,10]$. The purpose of introducing unified SINR is to make the SINR comparison between SU MIMO and MU MIMO schemes easier.

The system considered here has $n_{T}$ transmit antennas at the Base Station (BS) and $n_{R}^{i}$ receive antennas at the $i$ th Mobile Station (MS), $i=1,2, \cdots, K$. Without loss of generality, we assume that all the MSs have equal number of antennas $n_{R}$, and denote by $M=\min \left(n_{T}, n_{R}\right), N=\max \left(n_{T}, n_{R}\right)$.

In general, the received signal vector at the $i$ th MS can be modeled as

$$
\mathbf{y}_{i}=\mathbf{H}_{i} \sum_{i=1}^{K}\left(\mathbf{B}_{i} \mathbf{s}_{i}\right)+\mathbf{n}_{i}
$$


where $\mathbf{n}_{i} \in \mathbb{C}^{n_{R} \times 1}$ is a circularly symmetric complex Gaussian noise vector with zero mean and covariance matrix $N_{0} \mathbf{I} \in \mathbb{R}^{n_{R} \times n_{R}}$, i.e., $\mathbf{n}_{i} \sim \mathcal{C N}\left(\mathbf{0}, N_{0} \mathbf{I}\right) . \mathbf{H}_{i} \in \mathbb{C}^{n_{R} \times n_{T}}$ is the channel matrix between the BS and the $i$ th MS and $\mathbf{s}_{i} \in \mathbb{C}^{Q \times 1}$ is the transmit signal vector with elements picked from a unit energy constellation. $\mathbf{B}_{i} \in \mathbb{C}^{n_{T} \times Q}$ is the transmit precoding matrix for the $i$ th $\mathrm{MS}$, where $Q$ is the number of data streams. With a linear Minimum Mean Square Error (MMSE) receiver, also known as a Wiener filter, under the sum power constraint, the optimum precoding matrix can be expressed as $\mathbf{B}_{i}=\mathbf{U}_{i} \sqrt{\Sigma_{i}} \mathbf{V}_{i}$ [11]. Here $\mathbf{U}_{i}$ is an $n_{T} \times Q$ eigenvector matrix with columns corresponding to the $Q$ largest eigenvalues of the matrix $\mathbf{H}_{i} \mathbf{H}_{i}^{H}, \mathbf{H}_{i}^{H}$ is the Hermitian transpose of the channel matrix $\mathbf{H}_{i} \cdot \mathbf{V}_{i} \in \mathbb{C}^{Q \times Q}$ is an unitary matrix and $\boldsymbol{\Sigma}_{i}$ is a diagonal matrix with the $j$ th diagonal entry $\Sigma_{i}(j, j)$ represents the power allocated to the $j$ th established data substream, $j \in\{1,2, \cdots, Q\}$.

(1) can be further expressed as

$$
\mathbf{y}_{i}=\mathbf{H}_{i} \mathbf{B}_{i} \mathbf{s}_{i}+\mathbf{H}_{i} \sum_{j=1, i \neq j}^{K}\left(\mathbf{B}_{j} \mathbf{s}_{j}\right)+\mathbf{n}_{i}
$$

where the first item on the right hand side of (2) represents the desired received signal of the $i$ th MS. The second item is the MultiUser Interference (MUI) from the other active users. For the SU-MIMO SDM scheme of LTE downlink transmission, MUI can be eliminated by assigning users to distinct subcarrier groups, (i.e., PRBs) based on OFDMA. In this case, (2) can be simplified as

$$
\mathbf{y}_{i}=\mathbf{H}_{i} \mathbf{B}_{i} \mathbf{s}_{i}+\mathbf{n}_{i}
$$

which is equivalent to a single user MIMO system.

For the MU-MIMO SDM scheme of LTE downlink localized transmission, a special restriction is applied on the scheduler: Only the users with the same preferred precoding matricx $\mathbf{B}_{i}$ can be spatially multiplexed into one PRB, with users occupying different substreams [12] [6]. In this case, the received signal vector at the $i$ th MS can also be modeled as in (3). Different from the case in SU-MIMO transmission, the transmitted 
data vector $\mathbf{s}_{i}$ contains data from all users scheduled on the same PRB.

\section{SINR DISTRIBUTION FOR LINEARLY PRECODED SDM MIMO SCHEMES}

For a linearly precoded MIMO scheme using the linear MMSE receiver, the received $\operatorname{SINR}^{1}$ at the $j$ th spatial substream of the $i$ th MS, can be related to its Mean Square Error (MSE) as [11]

$$
\Gamma_{j}=M S E_{j}^{-1}-1, \quad j \in\{1,2, \cdots, Q\}
$$

In the above equation, for simplicity we have omitted the index for the $i$ th user.

For Schur-Concave objective functions, e.g., minimization of the weighted sum of the MSEs, the instantaneous SINR can be expressed as [11]

$$
\Gamma_{j}=\lambda_{j} \rho_{j}, \quad j \in\{1,2, \cdots, Q\}
$$

where $\lambda_{j}$ is the $j$ th non-zero largest eigenvalue of the matrix $\mathbf{H}_{i} \mathbf{H}_{i}^{H} \cdot \rho_{j}=p_{j} / N_{0}, p_{j}$ is the power allocated to the $j$ th established substream of the $i$ th MS and $N_{0}$ is the noise variance, see (1). It is well known that for Rayleigh MIMO fading channels, the complex matrix $\mathbf{H}_{i} \mathbf{H}_{i}^{H}$ is a complex central Wishart matrix [13].

The joint density function of the ordered eigenvalues of $\mathbf{H}_{i} \mathbf{H}_{i}^{H}$ can be expressed as [13]

$$
f_{\boldsymbol{\Lambda}}\left(\lambda_{1}, \cdots, \lambda_{M}\right)=\prod_{i=1}^{M} \frac{\lambda_{i}^{N-M}}{(M-i) !(N-i) !} \prod_{i<j}^{M-1}\left(\lambda_{i}-\lambda_{j}\right)^{2} \cdot \exp \left(-\sum_{i=1}^{M} \lambda_{i}\right),
$$

where $\lambda_{1} \geq \lambda_{2} \geq \cdots \geq \lambda_{M}$. For unordered eigenvalues, the joint density function can be obtained by $f_{\boldsymbol{\Lambda}}\left(\lambda_{1}, \cdots, \lambda_{M}\right) / M !$

\footnotetext{
${ }^{1}$ In the following, throughout this paper, we denote by an upper case letter a random variable and by the corresponding lower case letter its realization.
} 


\section{A. Linearly Precoded SDM SU-MIMO Schemes}

Now let us look at the joint density of the eigenvalues. The joint density function is needed to find the distribution of the unified effective SINR. For a linearly precoded spatial multiplexing MIMO scheme with 2 transmit antennas and 2 receive antennas, (6) can be simplified as

$$
f_{\boldsymbol{\Lambda}}\left(\lambda_{1}, \lambda_{2}\right)=\left(\lambda_{1}-\lambda_{2}\right)^{2} \exp \left(-\left(\lambda_{1}+\lambda_{2}\right)\right)
$$

With the Jacobian transformation [14], the joint density function of the SINRs of the two (assumed) established substreams is then given by

$$
f_{\boldsymbol{\Gamma}}\left(\gamma_{1}, \gamma_{2}\right)=\frac{1}{\rho_{1} \rho_{2}} f_{\boldsymbol{\Lambda}}\left(\lambda_{1} / \rho_{1}, \lambda_{2} / \rho_{2}\right)
$$

Let $x=1+\gamma_{1}$ and $y=1+\gamma_{2}$, then the unified effective SINR is given by $\Gamma_{e}=x y-1$, and the distribution function of $\Gamma_{e}$ can be expressed as

$$
F_{\Gamma_{e}}(\gamma)=\int_{-\infty}^{\infty} d x \int_{-\infty}^{\frac{\gamma+1}{x}} d y f_{\Gamma}(x-1, y-1)=\int_{-\infty}^{\infty} d x \int_{-\infty}^{\frac{\gamma+1}{x}} d y \frac{1}{\rho_{1} \rho_{2}} f_{\boldsymbol{\Lambda}}\left(\frac{x-1}{\rho_{1}}, \frac{y-1}{\rho_{2}}\right)
$$

By inserting (7) into (9), and limiting the integral region, we have

$$
F_{\Gamma_{e}}(\gamma)=\int_{1}^{\gamma+1} d x \int_{1}^{\frac{\gamma+1}{x}} d y \frac{1}{\left(\rho_{1} \rho_{2}\right)^{3}}\left(\rho_{2} x-\rho_{1} y+\rho_{1}-\rho_{2}\right)^{2} \exp \left(-\frac{1}{\rho_{1} \rho_{2}}\left(\rho_{2} x+\rho_{1} y-\rho_{1}-\rho_{2}\right)\right)
$$

(10) can be further expressed as

$$
\begin{aligned}
F_{\Gamma_{e}}(\gamma) & =\int_{0}^{\gamma} d v \int_{0}^{\frac{\gamma-v}{v+1}} d u \frac{1}{\left(\rho_{1} \rho_{2}\right)^{3}}\left(\rho_{2} v-\rho_{1} u\right)^{2} \exp \left(-\frac{1}{\rho_{1} \rho_{2}}\left(\rho_{2} v+\rho_{1} u\right)\right) \\
& =\int_{0}^{\gamma} d v \frac{1}{\left(\rho_{1} \rho_{2}\right)^{3}} \exp \left(-\frac{v}{\rho_{1}}\right) g(\gamma, v),
\end{aligned}
$$


where

$$
\begin{aligned}
g(\gamma, v) & =\rho_{2}{ }^{3} v^{2}\left[1-\exp \left(-\frac{\gamma-v}{\rho_{2}(v+1)}\right)\right]-2 \rho_{1} \rho_{2}{ }^{3} v\left[1-\exp \left(-\frac{\gamma-v}{\rho_{2}(v+1)}\right)\left(1+\frac{\gamma-v}{\rho_{2}(v+1)}\right)\right] \\
& +\rho_{1}{ }^{2} \rho_{2}{ }^{3}\left[2-\exp \left(-\frac{\gamma-v}{\rho_{2}(v+1)}\right)\left(\left(\frac{\gamma-v}{\rho_{2}(v+1)}\right)^{2}+\frac{2(\gamma-v)}{\rho_{2}(v+1)}+2\right)\right] .
\end{aligned}
$$

For localized downlink transmission with the SU-MIMO SDM scheme with linear precoding, applying the FD PF scheduling algorithm (as described previously) under the assumption of identical distribution of all effective SINR for all users, the probability that the SINR of a scheduled user is less than a certain reference SINR value i.e., the CDF of the post scheduling SINR per PRB can be computed by

$$
F_{\Gamma_{e}}^{M}(\gamma)=\left[P_{r}\left(\Gamma_{e} \leq \gamma\right)\right]^{K}=F_{\Gamma_{e}}(\gamma)^{K}
$$

where $K$ is the number of active users in the cell. (13) represents the distribution of the best user, i.e., the largest effective stream SINR among the $K$ users.

The PDF of the effective post scheduling SINR for the linearly precoded SDM SU-MIMO scheme can be obtained by differentiating its distribution function,

$$
\begin{aligned}
f_{\Gamma_{e}}^{M}(\gamma)= & K \int_{0}^{\gamma} \frac{1}{\left(\rho_{1} \rho_{2}\right)^{3}(1+v)} \exp \left(-\frac{v}{\rho_{1}}-\frac{\gamma-v}{\rho_{2}(1+v)}\right)\left(\rho_{2} v-\frac{\gamma-v}{1+v} \rho_{1}\right)^{2} d v \\
& \cdot\left(\int_{0}^{\gamma} \frac{1}{\left(\rho_{1} \rho_{2}\right)^{3}} \exp \left(-\frac{v}{\rho_{1}}\right) g(\gamma, v) d v\right)^{K-1} \cdot
\end{aligned}
$$

\section{B. Linearly Precoded SDM MU-MIMO Schemes}

Unlike SU-MIMO schemes where substreams are assigned to only one single user, in MU-MIMO schemes multiple users can be assigned to different substreams of each PRB, i.e., one substream per user. In order to calculate the unified effective SINR for the scheduled users per PRB, we need to find the distribution of the instantaneous SINR of each substream. This requires the derivation of the marginal Probability Density 
Function (PDF) of each eigenvalue. The marginal density function of the $k$ th ordered eigenvalue can be obtained by [15]

$$
f_{\Lambda_{k}}\left(\lambda_{k}\right)=\int_{\lambda_{k}}^{\infty} d \lambda_{k-1} \cdots \int_{\lambda_{2}}^{\infty} d \lambda_{1} \int_{0}^{\lambda_{k}} d \lambda_{k+1} \cdots \int_{0}^{\lambda_{M-1}} d \lambda_{M} f_{\Lambda}\left(\lambda_{1}, \cdots, \lambda_{M}\right)
$$

where $f_{\boldsymbol{\Lambda}}\left(\lambda_{1}, \cdots, \lambda_{M}\right)$ is given by (6).

A complicated expression of the distribution of the largest and the smallest eigenvalues can be found in the literature, e.g., $[16,17]$, but not for the other eigenvalues.

In [18], an approximated expression of the marginal PDF of eigenvalues is given as

$$
f_{\Lambda_{i}}\left(\lambda_{i}\right) \simeq \frac{1}{[\beta(i)-1] !} \frac{\lambda_{i}^{\beta(i)-1}}{\tilde{\lambda}_{i}^{\beta(i)}} \exp \left(-\lambda_{i} / \tilde{\lambda}_{i}\right)
$$

where $\beta(i)=\left(n_{T}-i+1\right)\left(n_{R}-i+1\right)$ and

$$
\tilde{\lambda}_{i}=\frac{1}{\beta(i)} \bar{\lambda}_{i}=\frac{1}{\beta(i)} \int_{0}^{\infty} \lambda_{i} f_{\Lambda}\left(\lambda_{i}\right) d \lambda_{i}
$$

It was verified by simulations in [18] that despite its simple form, (16) provides an accurate estimation of eigenvalues distribution of the complex central Wishart matrix $\mathbf{H H}^{H}$ for Rayleigh MIMO fading channel matrix $\mathbf{H}$.

Based on (5) and (16), the density function of the instantaneous SINR of the $i$ th substream can be expressed as

$$
f_{\Gamma_{i}}(\gamma)=\frac{1}{\rho_{i}} f_{\Lambda_{i}}\left(\gamma / \rho_{i}\right) \simeq \frac{1}{\rho_{i}} \frac{1}{[\beta(i)-1] !} \frac{\left(\gamma / \rho_{i}\right)^{\beta(i)-1}}{\tilde{\lambda}_{i}^{\beta(i)}} \exp \left(-\gamma /\left(\rho_{i} \tilde{\lambda}_{i}\right)\right)
$$

The outage probability, which is defined as the probability of the SINR being less than the targeted SINR within a specified time period, is a statistical measure of the system. From the definition, the outage proba- 
bility is simply the Cumulative Distribution Function (CDF) of the SINR evaluated at the targeted SINR. The outage probability can be obtained by

$$
\operatorname{Pr}\left(\Gamma_{i}<\gamma\right)=\int_{-\infty}^{\gamma} f_{\Gamma_{i}}(\alpha) d \alpha=\operatorname{Pr}\left(\lambda_{i}<\gamma / \rho_{i}\right) \simeq 1-\sum_{j=0}^{\beta(i)-1} \frac{\left(\gamma /\left(\rho_{i} \tilde{\lambda}_{i}\right)\right)^{j}}{j !} \exp \left(-\gamma /\left(\rho_{i} \tilde{\lambda}_{i}\right)\right)
$$

For LTE localized downlink transmission with a linearly precoded MU-MIMO scheme, we can schedule multiple users per PRB, i.e., one user per substream. Unlike the case of the MU-MIMO scheme without precoding described in [19], the instantaneous SINR of each substream is not identically distributed.

As shown earlier, the density function and the distribution function of the instantaneous SINR of each substream for single user case are given by (18) and (19), respectively. With multiple users case using the MU-MIMO SDM scheme and the FD PF packet scheduling algorithm, the distribution function of the instantaneous SINR for the $i$ th substream of each PRB can be obtained as

$$
F_{\Gamma_{i}}^{M}(\gamma)=\operatorname{Pr}\left(\Gamma_{1}<\gamma, \cdots, \Gamma_{K}<\gamma\right)=\prod_{k=1}^{K} \operatorname{Pr}\left(\Gamma_{i}<\gamma\right)=\left[F_{\Gamma_{i}}(\gamma)\right]^{K}
$$

Inserting (19) into (20), then we have

$$
F_{\Gamma_{i}}^{M}(\gamma) \simeq\left[1-\sum_{j=0}^{\beta(i)-1} \frac{\left(\gamma /\left(\rho_{i} \tilde{\lambda}_{i}\right)\right)^{j}}{j !} \exp \left(-\gamma /\left(\rho_{i} \tilde{\lambda}_{i}\right)\right)\right]^{K}
$$

The density function of the instantaneous SINR of the $i$ th substream of each PRB with linearly precoded MU-MIMO scheme using FD PF packet scheduling algorithm can then be obtained as

$f_{\Gamma_{i}}^{M}(\gamma)=\frac{d}{d \gamma} F_{\Gamma_{i}}^{M}(\gamma) \simeq \frac{K}{\rho_{i} \tilde{\lambda}_{i}} \frac{\left(\gamma /\left(\rho_{i} \tilde{\lambda}_{i}\right)\right)^{\beta(i)-1}}{(\beta(i)-1) !} \exp \left(-\frac{\gamma}{\rho_{i} \tilde{\lambda}_{i}}\right) \cdot\left[1-\sum_{j=0}^{\beta(i)-1} \frac{\left(\gamma /\left(\rho_{i} \tilde{\lambda}_{i}\right)\right)^{j}}{j !} \exp \left(-\frac{\gamma}{\rho_{i} \tilde{\lambda}_{i}}\right)\right]^{K-1}$

Note that for a dual substream linearly precoded SDM MU-MIMO scheme with FD PF packet scheduling algorithm, the distribution of instantaneous SINRs for the two substreams within a PRB is different. Con- 
sider the baseline antenna configuration of LTE, i.e., 2 antennas in both transmitter and receiver side. The distribution of the unified effective instantaneous SINR of the two substreams of each PRB for this case can be obtained by

$$
\operatorname{Pr}\left(\gamma_{u}<\gamma\right)=\int_{-\infty}^{\infty} f_{\Gamma_{1}}^{M}(x) F_{\Gamma_{2}}^{M}\left(\frac{\gamma-x}{x+1}\right) d x
$$

By inserting (21) and (22) into (23) and limiting the integral region, we have

$$
\begin{aligned}
\operatorname{Pr}\left(\gamma_{u}<\gamma\right) \simeq & \int_{0}^{\gamma} \frac{K}{\rho_{1} \tilde{\lambda}_{1}} \frac{\left(x /\left(\rho_{1} \tilde{\lambda}_{1}\right)\right)^{(\beta(1)-1)}}{(\beta(1)-1) !} \exp \left(-\frac{x}{\left(\rho_{1} \tilde{\lambda}_{1}\right)}\right)\left[1-\sum_{j=0}^{\beta(1)-1} \frac{\left(x /\left(\rho_{1} \tilde{\lambda}_{1}\right)\right)^{j}}{j !} \exp \left(-x /\left(\rho_{1} \tilde{\lambda}_{1}\right)\right)\right]^{K-1} \\
& \left.\cdot 1-\sum_{j=0}^{\beta(2)-1} \frac{\left((\gamma-x) /\left((x+1) \rho_{2} \tilde{\lambda}_{2}\right)\right)^{j}}{j !} \exp \left(-\frac{\gamma-x}{(x+1) \rho_{2} \tilde{\lambda}_{2}}\right)\right]^{K} d x
\end{aligned}
$$

Fig. 1 shows stream SINR distributions per PRB for single user dual stream MIMO and for LTE localized downlink transmission using precoded SDM MIMO schemes with baseline antenna configuration. The post scheduling multiuser effective SINR distributions for different scenarios are also shown. The number of users, i.e., the user diversity order, is 10 . These plots are obtained under the assumption of evenly allocated transmit power at the two transmitter antennas, and a transmitted signal to noise ratio equal to $20 \mathrm{~dB}$. For other parameters of the considered systems, we refer to, e.g., [6], [12].

It can be seen that for SU-MIMO scheme, the multiuser diversity gain (as shown in Fig. 1) at the 10th percentile of the post scheduled SINR per PRB is about $11 \mathrm{~dB}$ with 10 users. It is about $2 \mathrm{~dB}$ worse than the one for MU-MIMO scheme. This is due to the fact that SDM MU-MIMO can further exploit multiuser diversity in the spatial domain.

Fig. 2 shows the SINR distribution for systems with and without precoding. Compared with the system without precoding, the precoding scheme improves the SINR distribution for both of the investigated SDM schemes. At the 10th percentile of the post scheduled SINR, precoding gain for SU-MIMO scheme is about $3 \mathrm{~dB}$, while for MU-MIMO scheme, it is about $1.5 \mathrm{~dB}$. 


\section{CONCLUSION}

In this paper, we analyzed multiuser downlink SINR distribution for linearly precoded SDM MIMO schemes in conjunction with base station based packet scheduler. Two SDM multiuser MIMO schemes in the context of LTE downlink transmission were investigated, they are SU and MU MIMO schemes. In general, the outage probability for systems using SU-MIMO scheme is larger than the one with MU-MIMO scheme. Compared with the systems without precoding, linear precoding can improve the outage probability. Further works, such as the investigation of the average channel capacity [20] or the so called Shannon (Ergodic) Capacity [21], and the average symbol error rate per PRB, will be performed later. The above analysis can also be extended to other antenna configurations. 


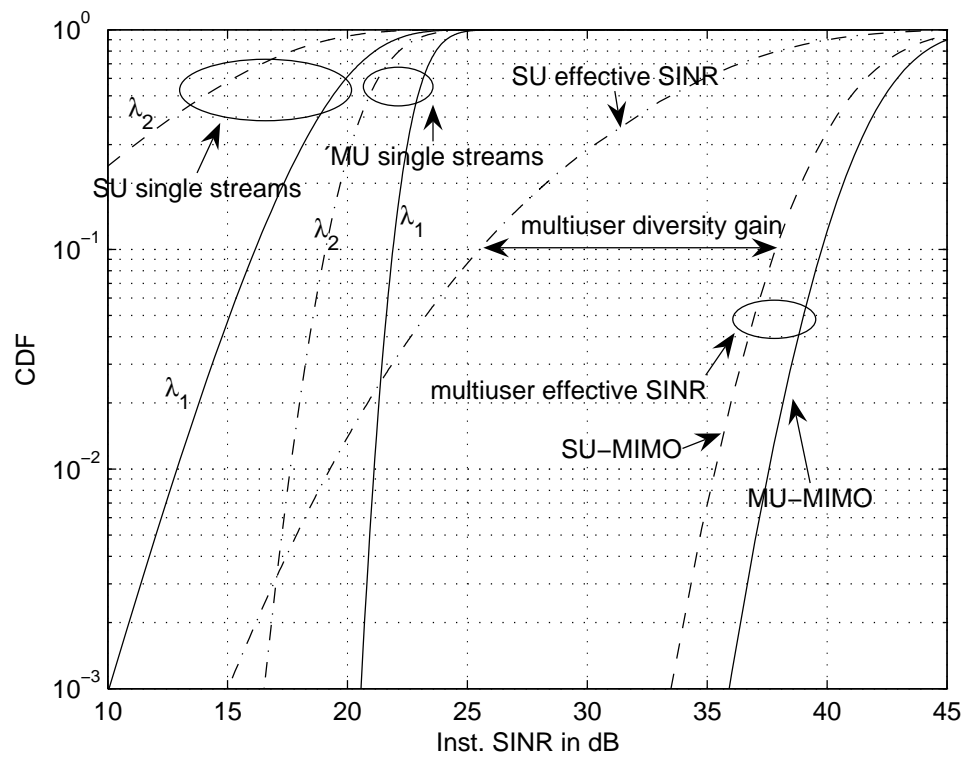

Fig. 1. SINR distribution for SDM linearly precoded multiuser SU and MU-MIMO schemes, number of users 10.

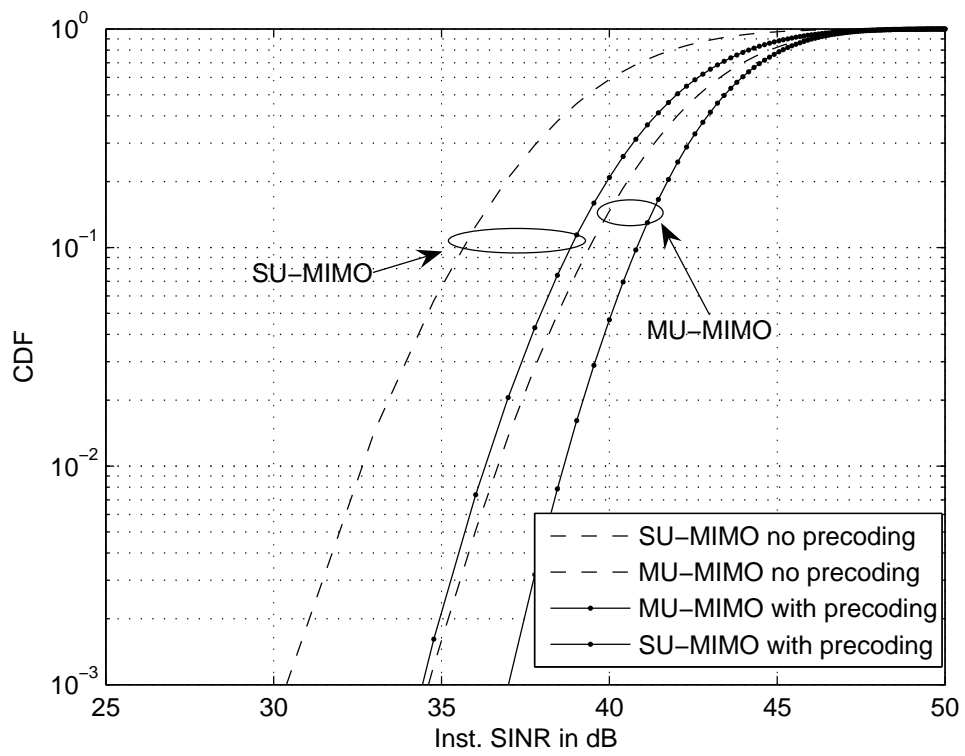

Fig. 2. SINR distribution for SDM multiuser MIMO schemes with and without precoding, number of users 10. 


\section{REFERENCES}

[1] J. Zhang, Y. Wu, M. Xu, and J. Wang, "Linear transmitter precoding design for downlink of multiuser MIMO system," Electronics Letters, vol. 41 , no. 14 , July 2005 .

[2] Q. H. Spencer, A. L. Swindlehurst, and M. Haardt, "Zero-forcing Methods for Downlink Spatial Multiplexing in Multiuser MIMO Channels," Transactions on Singal Processing, vol. 52, no. 2, pp. 461-471, Feb. 2004.

[3] A. G. Kogiantis, N. Joshi, and O. Sunay, "On Transmit Diversity and Scheduling in Wireless Packet Data," in IEEE ICC, 2001, pp. 2433-2437.

[4] L. T. Berger, T. E. Kolding, J. R. Moreno, P. Ameigeiras, L. Schumacher, and P. E. Mgensen, "Interaction of transmit diversity and proportional fair scheduling," in IEEE VTC, spring 2003, pp. 2423-2427.

[5] R. Gozali, R. M. Buehrer, and B. D. Woerner, "The impact of multiuer diversity on space time block coding," IEEE Commun. Letters, vol. 7, no. 5, pp. 213-215, May 2003.

[6] 3GPP TR 25.814 V7.0.0, "Physical Layer Aspects for Evolved UTRA,” Tech. Rep., June 2006.

[7] T. Park, O.-S. Shin, and K. B. Lee, "Proportional Fair Scheduling for Wireless Communication with Multiple Transmit and Receive Antennas," in IEEE VTC, Florida, USA, October 2003, vol. 3, pp. 1573 - 1577.

[8] J. M. Holtzman, "CDMA forward link waterfilling power control," in IEEE Vehicular Technology Conference (VTC), May 2000, pp. 1636-1667, Toyko, Japan.

[9] J. R. Fonollosa, Ed., Design of the Single user Optimal Transmit and Receive, Number D3.1. IST-027187-SURFACE, Sept. 2006.

[10] N. Wei, T. B. Sørensen, T. E. Kolding, and P. E. Mogensen, "Analysis and Evalution of Link Adaptation with MIMO adaptation,” in IEEE VTC, Sept. 2006, pp. 1-5.

[11] D. P. Palomar, J. M. Cioffi, and M. A. Lagunas, "Joint Tx-Rx Beamforming Design for Multicarrier MIMO Channels: A Unified Framework for Convex Optimization,” IEEE Transaction on Signal Processing, vol. 51, no. 9, pp. 2381-2401, Sept. 2003.

[12] N. Wei, A. Pokhariyal, T. B. Sørensen, T. E. Kolding, and P. E. Mogensen, "Performance of MIMO with Frequency Domain Packet Scheduling in UTRAN LTE downlink,” in IEEE VTC, Apr. 2007, pp. 1177-1181.

[13] E. Edelman, "Eigenvalues and condition numbers of random matrices," SLAM J. Matrix Anal. Appl., vol. 9, no. 4, pp. 543-559, Oct. 1988.

[14] L. G. Alberto, Probability and Random Processes for Electrical Engineering, Addison-Wesley Publishing Company, 2 nd edition, 1994.

[15] M. Wemmstrom, On MIMO Systems and Adaptive Arrays for Wireless Communication: Analysis and Practical Issues, Ph.D. thesis, Uppsala University, 2002.

[16] T. Sugiyama, "On the distribution of the largest latent root of the covariance matrix," Ann. Math. Statist., vol. 38, pp. $1148-1151,1967$.

[17] T. C. Cheng P. R. Krishnaiah, "On the exact distribution of the smallest roots of the Wishart matrix using zonal polynomials," Ann. Math. Statist., vol. 23, pp. 293-295, 1967.

[18] T. Taniguchi, S. Sha, and Y. Karasawa, "Statistical Distribution of Eigenvalues of Correlation Matrices in i.i.d MIMO Channels under Rayleigh Fading," in IEEE International Symposium on Personal, Indoor and Mobile Radio Communications, 2005, pp. 537-541.

[19] J. R. Fonollosa, Ed., Design of the Multiuser Optimal Transmit and Receive Strategy, Number D4.1. IST-027187-SURFACE, Dec. 2006.

[20] W. C. Y. Lee, "Estimate of channel capacity in Rayleigh fading enviroment," IEEE Trans. on Veh. Technol., vol. 39, pp. 187-190, Aug. 1990. 
[21] A. Goldsmith, Wireless Communications, Cambridge University Press, 2005. 